\title{
NORMAL AND ABNORMAL HUMAN FACE DETECTION BASED ON DCT AND FFT TECHNIQUES- A PROPOSED METHOD
}

\author{
Payal Bose1, Prof. Shawni Dutta2, Prof. Vishal Goyal 3 and Prof. Samir K. Bandyopadhyay4,* \\ ${ }_{1}$ Research Scholar, Lincoln University College, Kota Bharu, Kelantan, Malaysia \\ ${ }_{2}$ Department of Computer Science, The Bhawanipur Education Society College, Kolkata, India \\ 3GLA University, Mathura-Delhi Road Mathura, Chaumuhan, Uttar Pradesh, India \\ 4Distinguish Professor, Lincoln University College, Kota Bharu, Kelantan, Malaysia \\ * Corresponding author. (e-mail address:1954samir@gmail.com)
}

\begin{abstract}
In today's world face detection is the most important task. Due to the chromosomes disorder sometimes a human face suffers from different abnormalities. For example, one eye is bigger than the other, cliff face, different chinlength, variation of nose length, length or width of lips are different, etc. For computer vision currently this is a challenging task to detect normal and abnormal face and facial parts from an input image. In this research paper a method is proposed that can detect normal or abnormal faces from a frontal input image. This method used Fast Fourier Transformation (FFT) and Discrete Cosine Transformation of frequency domain and spatial domain analysis to detect those faces.
\end{abstract}

Keyword. Face Detection, Euclidean Distance, Fast Fourier Transformation, Discrete Cosine Transformation, Facial Parts Detection, Frequency domain, Spatial domain.

\section{INTRODUCTION}

A face is a key feature to identify a human. During the early stage of embryogenesis development genetic factors playing a key role to develop a face. Due to the chromosomal disorder, facial abnormalities can occur. In this modern era with advanced research technologies, it is possible to identify this facial disorder with help of various methods. Researchers develop various methods to identify a face. They also develop algorithms to detect different facial parts. The primary objective of this study is to detect whether a human face is normal or abnormal. To implement this process in this research first face and facial detection system are developed. Then based on the length and the width of the face and facial areas the detection process is developed. This research is a real-world application with a high accuracy rate. The Fast Fourier transformation and Discrete Cosine transformation are used for the precise detection of normal and abnormal humans. The findings of this research suggested that this proposed methodology is useful in this research area and can lead more objective research in the future.

\section{RELATED WORKS}

Several researchers implemented several algorithms for face detection with different classification algorithms. For this, they use either a single classification model or a combined model. To detect a face a faked or not researchers used Generative Adversarial Networks (GANs) to automatically detected those fake faces [1]. Some researchers review in their paper about the Down syndrome (DS) disorder of the human, caused by chromosome abnormalities. They describe face detection, feature extraction, and different classification techniques on it [2]. To detect a face researchers used haar cascade likes and Euclidean distance measurement in their experiment. They show that the recognition rate is $91.1 \%$ for the experiment [3]. Researchers proposed a generalized solution to detect visually observable symptoms on faces. For this purpose they used a semi-supervised learning technique with different classification technique algorithms [4]. Other researchers proposed in their research paper an improved technique in 
face recognition based on Principal Component Analysis and Fast Fourier transformation algorithm. They used the yale database and series of experiments to develop the model [5].

\section{HUMAN FACE STRUCTURE}

Each human face has unique features aspects of every individual. In the human body, the face is the key body part for identifying a human. In anatomy human face [6] is divided into three main regions, 1) Upper Face, 2) Middle face and 3) Lower face.

\subsection{Upper Face}

The area started from just below the hairline to the under lower eye-lid, is known as the upper face. This region is divided into two regions.

Forehead. It is the biggest and superior region of the upper face area. This area started from just below the hairline area. It contains two features, skull, and scalp.

Eyes. In the upper face region, the eyes are situated at the semi-circular orbital socket. It is the other superior area of the upper face region. It contains two eye-lids, upper and lower eyelids, and the eyebrows.

\subsection{Middle Face}

The middle face started from the lower eyelid region to just above the upper lip region. This is the central part of a face. It is divided into three parts 1) Nose, 2) Cheek, 3) Ear.

Nose. It is the middle line structure that extends to the face region. In this experiment the nose region divides into two parts, 1) Nose tip and 2) The region from the nose tip to just above the upper lip region.

Cheeks. This region is lateral to the nose. It contains skin and fat pads.

Ears. Humans' ears are used to hear the sound from the environment. This part is the lateral outline of the nose region.

\subsection{Lower Face}

The lower face region is started from the upper lip region to the ching region. This part contains four parts 1)Upper Lip region, 2)Lip region, 3)Chin region, and 4) Jawline region.

Upper Lip Region. This area started from the below nose region to just above the lip region.

Lip Region. This region is started from just below the upper lip region to just above the chin region. This area is also known as the mouth.

Chin Region. This area started from the below lip region to the end of the face region. This is the expanded region of the cheeks.

Jaw Lines. This area is started with the parallel to the nose tip point. The jawline region defines the lower structure of the human face.

All the regions' details described above are shown in figure 1. All the yellow points with red color encircled are the main 8 points of a face and others are used to define those areas more precisely. 


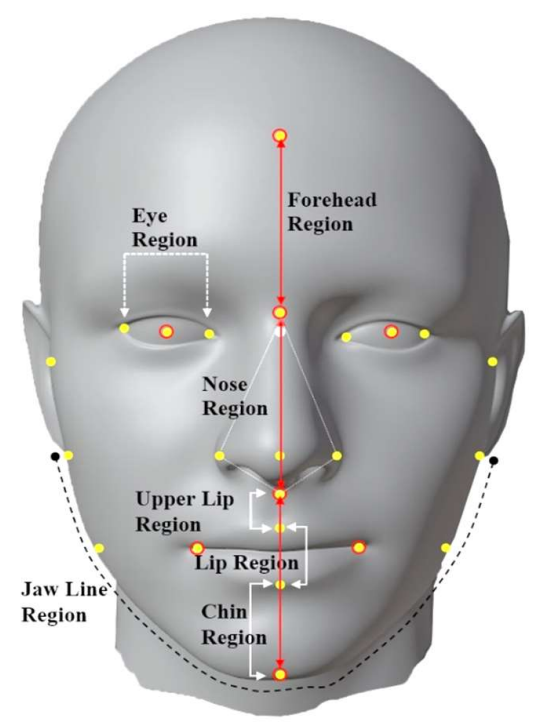

Figure 1: Human Face Structure

\section{PROPOSED METHODOLOGY}

The proposed method includes six steps. They are 1) Pre-Processing, 2) Face Detection, 3) Facial Parts Detection, 4)Euclidean distance calculation, 5) Fast Fourier and Discrete Cosine Transformation, and 6) Classification analysis. The steps are shown in figure 2.

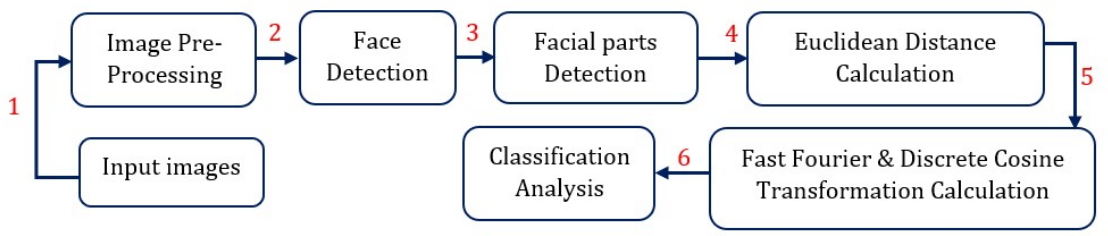

Figure 2: Steps Involved In The Proposed Method

\subsection{Image Pre-Processing}

In this part, the below steps are followed [7-9].

Step 1: Resize the input image.

Step 2: Convert the RGB input color image into grayscale image.

Step 3: To remove any noise from the image apply a filter.

Step 4: To improve the contrast of the filtered image apply the histogram equalization technique.

In this experiment to remove the noise from the grayscale image Gaussian filter is used.

\subsection{Face Detection}

In this experiment to detect a normal or an abnormal human, face detection is the key step. After pre-processing the input image this is the second step. Here to achieve this the Viola-Jones face detection algorithm [10] is used. This algorithm is very much useful to detect and categorize a human from non-human faces. The accuracy to detect a face using this algorithm is much higher.

\subsection{Facial Parts Detection}

The third step after detecting a face from an image is to detect the different facial parts of that face [11]. In this step different facial features like forehead, nose, both eye regions, upper lip, lip, chin area, and jawline detection are involved. For detecting these facial parts the Viola-Jones algorithm is used. 


\subsection{Euclidean Distance Calculation}

After successfully detecting the face and different facial parts, the fourth step is to calculate the length and width of those regions. To calculate the length and width of those extracted facial features the Euclidean Distance [12] measurement formula shown in equation 3 is used.

Euclidean Distance, $E D=\sqrt{\left(x_{2}{ }^{2}-x_{1}{ }^{2}\right)-\left(y_{2}{ }^{2}-y_{1}{ }^{2}\right)}$

Where $\left(x_{1}, y_{1}\right)$ and $\left(x_{2}, y_{2}\right)$ are the two co-ordinates of two points.

\subsection{Fast Fourier Transformation (FFT) and Discrete Cosine Transformation (DCT)}

The fifth step is to calculate the fast Fourier transformation and Discrete Cosine transformation of the created matrices from step four.

Fast Fourier Transformation (FFT). Fast Fourier transformation (FFT) is part of the Discrete Fourier transformation (DFT) algorithm. This algorithm is used to reduce the number of computations for $\mathrm{N}$ points. It reduces the computations of $\mathrm{N}$ points to $2 n \lg N$ from $2 N$. Where $\boldsymbol{l g}$ is the algorithm of base- 2 . FFT has two classes 1) decimation in time and 2) decimation in frequency. The basic idea behind FFT is to break the N points into two $\frac{N}{2}$ lengths. The equation of FFT is shown in equation 2. [13]

$$
\begin{aligned}
& \sum_{n=0}^{N-1} a_{n} e^{-\frac{2 \pi i n k}{N}}=\sum_{n=0}^{\frac{N}{2}-1} a_{2 n} e^{-\frac{2 \pi i(2 n) k}{N}}+\sum_{n=0}^{\frac{N}{2}-1} a_{2 n+1} e^{-\frac{2 \pi i(2 n+1) k}{N}} \\
& =\sum_{\mathrm{n}=0}^{\frac{N}{2}-1} \mathrm{a}_{\mathrm{n}} \text { even }^{-\frac{2 \pi \mathrm{ink}}{\frac{N}{2}}}+\mathrm{e}^{-2 \pi \mathrm{in} / \mathrm{N}} \sum_{\mathrm{n}=0}^{\frac{N}{2}-1} \mathrm{a}_{\mathrm{n}} \text { odd }_{\mathrm{e}} \mathrm{e}^{-\frac{2 \pi \mathrm{ink}}{\frac{N}{2}}}
\end{aligned}
$$

Discrete Cosine Transformation (DCT). The Discrete Cosine transformation technique [14] is used to convert a signal into its frequency components. In this experiment, this technique was used to convert the pixel values in the spatial domain to its frequency domain. The equation of DCT is shown in equation 3.

$$
F(\mathrm{i}, \mathrm{j})=\frac{1}{4} \mathrm{C}_{\mathrm{i}} \mathrm{C}_{\mathrm{j}} \sum_{\mathrm{x}=0}^{7} \sum_{\mathrm{y}=0}^{7} \mathrm{f}(\mathrm{i}, \mathrm{j}) \cos \frac{(2 x+1) \mathrm{j} \pi}{16} \cos \frac{(2 y+1) \mathrm{i} \pi}{16}
$$

\subsection{Classification Analysis}

The final step is to compare the calculated matrices of the training dataset with the test dataset. Based on the threshold value, a binary matrix is created. This matrice is used to determine the normal and abnormal humans from the input facial images. The required algorithms and data flow diagrams are shown in the algorithms and data flow diagram section.

\section{ALGORITHMS AND DATA FLOW DIAGRAM}

The algorithms that describe the proposed methodology are given below.

Detection_of_normal_abnormal_humans(Image dataset) [Perform the normal and abnormal human detection] 


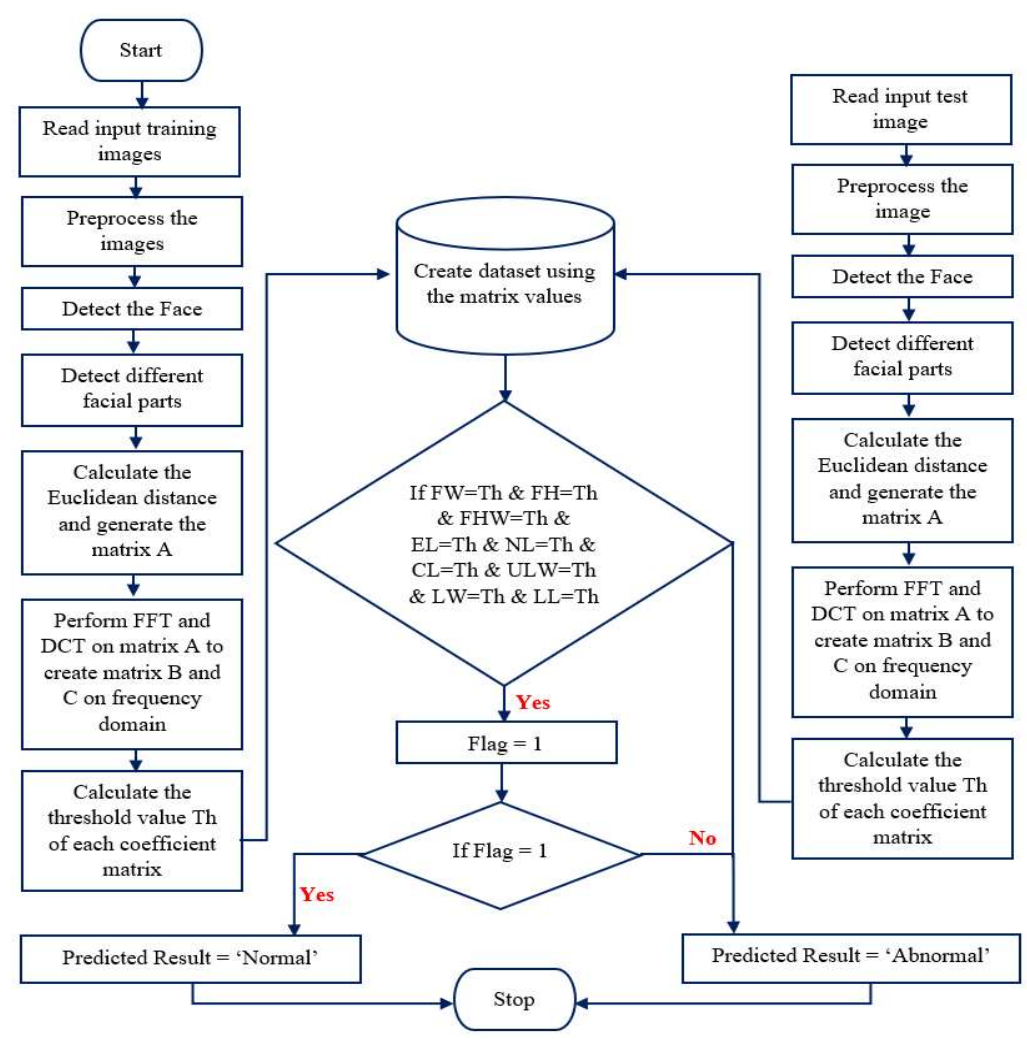

Figure 3: Data Flow Diagram of Proposed Method

\section{RESULTS}

In this experiment the input database contains more than 2000 images of male and female humans of different ages. This database made primarily created by us. In addition in this experiment, the World Wide Web (WWW) repository is used for requires images to create the primary dataset.

In this image dataset, all the images are in the RGB version. The proposed algorithm was implemented with the help of MATLAB 2020a. From preprocessing to face and different facial features detection several functions in MATLAB are used. The general structure of a human face and its different facial parts with their distance measurements are described in figure 4. 


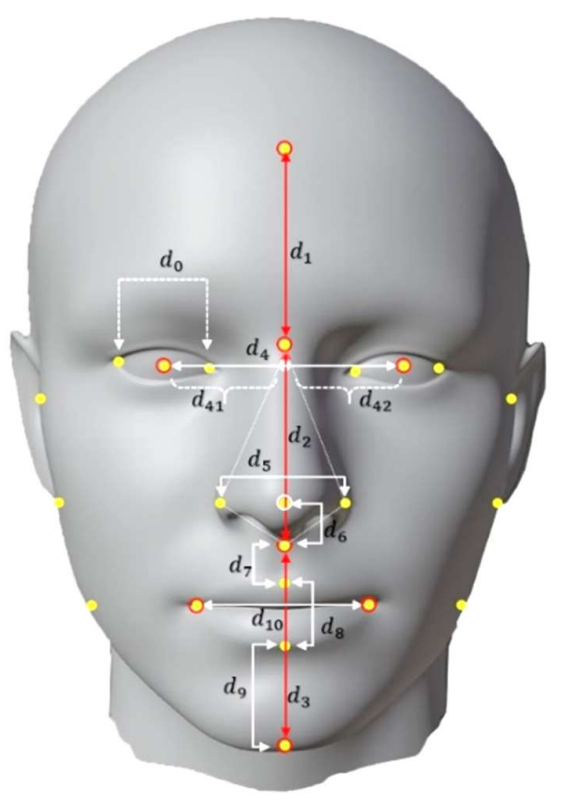

Figure 4: The General Diagram of Human Face and Its Different Facial Parts

In figure 4 the yellow dots with red circles are the main points of a human face structure. All other yellow points are used to define the complete face structure. The yellow point with the white circle defines the top tip point of the nose and the high point of a human face. The length $d_{0}$ to $d_{10}$ defines the Euclidean distance length of different facial parts.

\begin{tabular}{cc}
\hline Points & Description (Euclidean Distance) [Based on Figure 1, 4] \\
\hline$d_{0}$ & Distance between two eye points (EL) \\
$d_{1}+d_{2}+d_{3}$ & Face Length (FL) \\
$d_{2}$ & Nose Length (NL) \\
$d_{4}\left(d_{41}+d_{42}\right)$ & Distance between the center point of the left eye to the right eye \\
$d_{5}$ & Distance Between Nose tip point to the endpoint of the nose region \\
$d_{6}$ & Width of Upper Lip (ULW) \\
$d_{7}$ & Width of Lip Region (LW) \\
$d_{8}$ & Width of Chin region (CL) \\
$d_{9}$ & Length of Lip region (LL) \\
$d_{10}$ &
\end{tabular}

The preprocessing result of the human face is depicted in figure 5. And detecting different facial parts depicted in figure 6. 


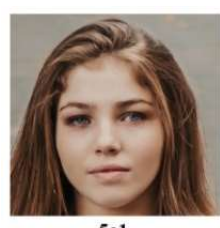

5a1

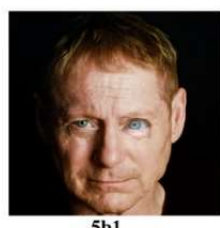

$5 b 1$

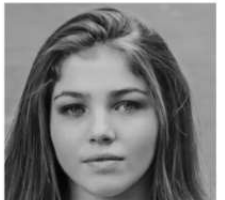

$5 \mathrm{a} 2$

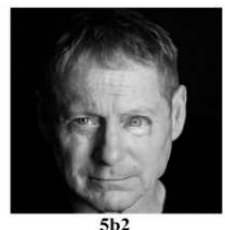

$5 b^{2}$

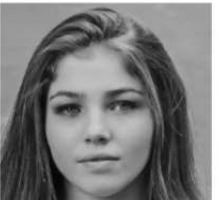

5,3

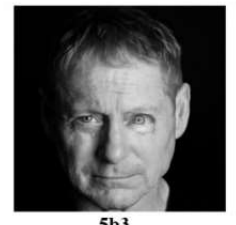



Normal

Human Face

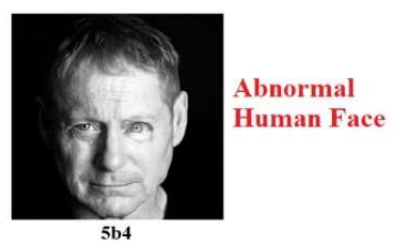

Figure 5: The Preprocessing Result [(a1,b1) Original Image, (a2,b2) Grayscale Image, (a3,b3) noise removed using Gaussian Filter, (a4,b4) Histogram Equalized Image]



Figure 6: Different Facial Part Detection and Their Euclidean Distance Calculation Area [(6a,6d) Original Preprocessed image, (6b,6e) Detected Facil Parts Region, (6c,6f) Different Facial Parts] 


\section{CONCLUSION}

Normal and Abnormal human detection is one of the secondary stages of human identification. In this experiment the proffered technique determines a human is normal or not based on their facial features. At the first stage, this technique successfully detected the human face and their facial features from input images. Then in the second stage, it calculated the length and width of those facial features. Finally based on this length and width this technique can determine whether the face is normal or abnormal.

\section{REFERENCES}

[1] Tariq, S., Lee, S., Kim, H., Shin, Y., \& Woo, S. S. (2018). Detecting both machine and human created fake face images in the wild. Proceedings of the ACM Conference on Computer and Communications Security, March 2020, 81-87. https://doi.org/10.1145/3267357.3267367

[2] Agbolade, O., Nazri, A., Yaakob, R., Ghani, A. A., \& Cheah, Y. K. (2020). Down syndrome face recognition: A review. Symmetry, 12(7), 1-17. https://doi.org/10.3390/sym12071182

[3] Wu, H., Cao, Y., Wei, H., \& Tian, Z. (2021). Face Recognition Based on Haar like and Euclidean Distance. Journal of Physics: Conference Series, 1813(1). https://doi.org/10.1088/1742-6596/1813/1/012036

[4] Wang, K., \& Luo, J. (2016). Detecting Visually Observable Disease Symptoms from Faces. Eurasip Journal on Bioinformatics and Systems Biology, 2016(1). https://doi.org/10.1186/s13637-016-0048-7

[5] Dehai, Z., Da, D., Jin, L., \& Qing, L. (2013). A PCA-based Face Recognition Method by Applying Fast Fourier Transform in Pre-processing. Proceedings of 3rd International Conference on Multimedia Technology(ICMT-13), 84, 1155-1162. https://doi.org/10.2991/icmt-13.2013.141

[6] Nguyen JD, Duong H. Anatomy, Head and Neck, Face. [Updated 2020 Jul 27]. In: StatPearls [Internet]. Treasure Island (FL): StatPearls Publishing; 2021 Jan-. Available from: https://www.ncbi.nlm.nih.gov/ books/NBK551530/

[7] Talebi, H., \& Milanfar, P. (2021). Learning to Resize Images for Computer Vision Tasks. 1. http://arxiv.org/abs/2103.09950

[8] Singhal, P., Verma, A., \& Garg, A. (2017). A study in finding effectiveness of Gaussian blur filter over bilateral filter in natural scenes for graph based image segmentation. 2017 4th International Conference on Advanced Computing and Communication Systems, ICACCS 2017, i, 4-9. https://doi.org/10.1109/ICACCS.2017.8014612

[9] Patel, O., P. S. Maravi, Y., \& Sharma, S. (2013). A Comparative Study of Histogram Equalization Based Image Enhancement Techniques for Brightness Preservation and Contrast Enhancement. Signal \& Image Processing: An International Journal, 4(5), 11-25. https://doi.org/10.5121/sipij.2013.4502

[10] Wang, Yi-Qing. (2014). An Analysis of the Viola-Jones Face Detection Algorithm. Image Processing On Line. 4. 128-148. 10.5201/ipol.2014.104.

[11] Vikram, K., \& Padmavathi, S. (2017). Facial parts detection using Viola Jones algorithm. 2017 4th International Conference on Advanced Computing and Communication Systems, ICACCS 2017, 2015-2018. https://doi.org/10.1109/ICACCS.2017.8014636

[12] Hassanpour, Hamid (2021): Euclidean Distance Filter for Image Processing. TechRxiv. Preprint. https://doi.org/10.36227/techrxiv.13664615.v1

[13] Oberst, U. (2007). The fast Fourier transform. SIAM Journal on Control and Optimization, 46(2), 496-540. https://doi.org/10.1137/060658242

[14] Bhattacharjee, P. K., \& Bengal, M. W. (2020). Comparison of Discrete Fourier Transform and Fast Fourier Transform with Reduced Number of Multiplication and Addition Operations. International Journal of Applied Mathematics and Informatics, Volume 14(February). https://doi.org/10.46300/91014.2020.14.16 